\title{
Actin-granule formation is an additional step in cardiac myofibroblast differentiation
}

\author{
Li He $^{1 \#}$, Ruiqi Liu ${ }^{2 \#}$, Honghua Yue ${ }^{1}$, Shuofang Ren ${ }^{1}$, Guonian Zhu ${ }^{3}$, Yingqiang Guo ${ }^{1}$, Chaoyi Qin ${ }^{1}$ \\ ${ }^{1}$ Department of Cardiovascular Surgery, West China Hospital, Sichuan University, Chengdu, China; ${ }^{2}$ Department of Burn and Plastic Surgery, West \\ China Hospital, Sichuan University, Chengdu, China; ${ }^{3}$ Research Core Facility of West China Hospital, Sichuan University, Chengdu, China \\ Contributions: (I) Conception and design: L He; (II) Administrative support: Y Guo; (III) Provision of study materials or patients: C Qin; (IV) \\ Collection and assembly of data: R Liu; (V) Data analysis and interpretation: G Zhu; (VI) Manuscript writing: All authors; (VII) Final approval of \\ manuscript: All authors. \\ \#These authors contributed equally to this work. \\ Correspondence to: Chaoyi Qin, MD. Department of Cardiovascular Surgery, West China Hospital, Sichuan University, 36th Guoxue Road, Chengdu \\ 610041, China. Email: qinchaoyi@wchscu.cn.
}

\begin{abstract}
Background: Atrial fibrillation is the most common and long-lasting cardiac arrhythmia, and profoundly effects the daily lives of patients. The pathogenesis and persistence of atrial fibrillation is closely related to the cardiac fibroblast and its myofibroblast differentiation as increased collagen synthesis and migration capability. Thus better understanding of myofibroblast differentiation is essential for the prevention and treatment of atrial fibrillation
\end{abstract}

Methods: Cardiac fibroblasts were isolated from neonatal rats and its actin structure was analyzed by immunofluorescence staining. Myofibroblast differentiation was induced by Angiotensin II (Ang II) and ROCK signaling related proteins were determined by western blot. Fasudil and Ricolinostat were employed to abrogate ROCK signaling and their effects on myofibroblast differentiation were assessed by IF microscopy and Celigo Image Cytometry.

Results: Stress actin fibers similar to actin filaments in myofibroblast differentiation are regulated by ROCK signaling, and our results also suggested Guanine nucleotide exchange factor-H1 (GEF-H1) phosphorylation could be induced by Ang II. In addition, Fasudil could down-regulate RhoA, GEF-H1, and phosphorylated GEF-H1 to inhibit ROCK signaling and further reduce Col I expression and the myofibroblast proportion.

Conclusions: An individual phase characterized by actin-granule formation was identified in cardiac myofibroblast differentiation. In the meanwhile, myofibroblast differentiation and its F-actin assembly could be detained in this phase by Fasudil abrogating the ROCK signaling pathway.

Keywords: Fibrosis; myofibroblast differentiation; stress actin fiber; actin-granule; ROCK

Submitted Dec 10, 2020. Accepted for publication Jan 21, 2021.

doi: 10.21037/atm-20-8231

View this article at: http://dx.doi.org/10.21037/atm-20-8231

\section{Introduction}

Atrial fibrillation (AF) is the most common cardiac arrhythmia and raises concerns because of its fatal complications and rising age incidence (1-3). Research on the mechanisms of atrial fibrillation pathogenesis and persistence has shown pathological fibrosis and cardiac fibroblasts have an important role in initiating and sustaining cardiac remodeling in $\mathrm{AF}$ progression. The differentiation of cardiac fibroblasts to myofibroblasts has been the subject of much investigation into atrial fibrillation related fibrosis and has been identified as a consequence of the upregulated expression of Collagen $\mathrm{I}(\mathrm{Col} \mathrm{I})$ and TGF- $\beta 1$ (transforming growth factor- $\beta 1$ ) accompanied with Alpha-smooth muscle actin ( $\alpha$-SMA) 
synthesis and assembly (4).

Angiotensin II (Ang II) has been identified as a potent factor which initiates $\mathrm{AF}$ related fibrosis and myofibroblast differentiation $(5,6)$. It is mainly through an angiotensin receptor type 1 that Ang II activates the JNK/STAT signaling pathway to induce myofibroblast differentiation and the secretion of TGF- $\beta 1$ along with other profibrotic cytokines. This in turn activates smad2/3 signaling and further promotes fibrosis $(7,8)$. Aoki et al. and Barnes et al. showed that Ang II also gives rise to stress actin fiber assembly in cardiac myocytes, suggesting a possible correlation between the microfilament remodeling of myofibroblasts and stress fiber assembly (9-11).

Stress actin fiber formed in non-muscle cells will increase their capacity of contraction and migration, in which the activation of ROCK signaling is essential (12). As serine/ threonine kinases, ROCK1 and ROCK 2 are the main effectors of ROCK signaling and are activated by RhoA ROCK signaling related GTPase. Indirect evidence that supports the correlation of myofibroblast differentiation and stress actin fibers lies in the cross-regulation of different cytoskeleton components. This is because histone deacetylase 6 (HDAC6) regulation of the balance of acetylation and deacetylation of $\alpha$-tubulin has been proven to be highly relevant to atrial fibrillation related microfilament remodeling, which could increase the release of tubulinbound Guanine nucleotide exchange factor-H1 (GEF-H1) to activate ROCK signaling subsequently (13-16).

While the definition of myofibroblast differentiation incorporates $\alpha$-SMA filament formation and fibrosis upregulation, the relationship between the two remains unclear. During our research on the effects of specific pathogens on cardiac fibroblasts, we found that despite low collagen levels, in many fibroblasts $\alpha$-SMA was expressed. However instead of direct assembly, these monomers were condensed into a perinuclear region spared by the rearrangement of the vimentin (intermediate filament) network. Since fibroblasts were present, we presumed this to be an individual phase of cardiac fibroblast development in the early period of differentiation. This caused us to question whether $\alpha$-SMA synthesis and the assembly of myofibroblast differentiation is relevant to the simultaneous upregulation of Collagen I and TGF- $\beta 1$, or programmed in the process ahead of it. To date, neither the involvement of ROCK signaling in myofibroblast differentiation nor the relationship of its $\alpha$-SMA and pro-fibrotic transition have been elucidated.
We present the following article in accordance with the ARRIVE reporting checklist (available at http://dx.doi. org/10.21037/atm-20-8231).

\section{Methods}

\section{Ethical statement}

Animal protocols were approved and animal experiments were supervised by Animal Ethics Committee of West China Hospital and the Research Core Facility of West China Hospital, in compliance with the guidelines of Ethics Committee of West China Hospital for the care and use of animals.

\section{Experimental animals}

We purchased 1-day old neonatal SD rats from the Dashuo Biomedical Technique Company.

\section{Fibroblasts isolation and culture}

Cardiac fibroblasts were isolated from the hearts of the neonatal SD rats. The harvested hearts were cut into patches of $1 \mathrm{~mm}^{3}$ then repeatedly digested in 3-5 $\mathrm{mL}$ Phosphate Buffer Saline (PBS) containing 1\% trypsin (Gibco, USA) and collagenase II (Gibco, USA) at $37^{\circ} \mathrm{C}$ for $10 \mathrm{~min}$. This was stopped by transferring the supernatants to an equal volume of Dulbecco's modified eagle medium (DMEM) (Gibco, USA) with 10\% fetal bovine serum (FBS) (Gibco, Australia). The cell suspension was centrifuged at 1,000 rpm for $10 \mathrm{~min}$. and cells were re-suspended in a $4 \mathrm{~mL}$ medium, from which the fibroblasts were purified by 1.5 -hour differential adherence. The obtained fibroblasts were cultured in DMEM containing 10\% FBS and 1\% penicillinstreptomycin antibiotic solution (Gibco, USA) at $37^{\circ} \mathrm{C}$ and $5 \% \mathrm{CO}_{2}$.

\section{Fibroblasts treatment}

Before any treatment, all cells were serum starved for 24 hours. Ang II (Harveybio, China) was added reaching a concentration of $10 \mu \mathrm{mol} / \mathrm{mL}$. At the same time, $5 \mu \mathrm{M}$ Ricolinostat and $50 \mu \mathrm{M}$ Fasudil were adopted. All treatments lasted 24 hours and for co-incubation, Ricolinostat or Fasudil were added 6 hours before the addition of Ang II. 


\section{Immunofluorescence staining and microscopy}

Cells were fixed with $4 \%$ paraformaldehyde for $15 \mathrm{~min}$, followed by permeabilizing with $0.5 \%$ Triton-X-100 (Biofroxx, German) for $10 \mathrm{~min}$ and sealing with corresponding serum (Solarbio, China). For the identification of fibroblasts, the primary antibodies included rabbit monoclonal [EPR3776] to vimentin (Abcam, UK, ab92547), mouse monoclonal [1A4] to alpha smooth muscle Actin (Abcam, UK, ab7817), and rabbit monoclonal [E184] to alpha smooth muscle Actin (Abcam, UK, ab32575). The involved secondary antibodies in all included A488conjugated donkey anti-rabbit $\mathrm{IgG}(\mathrm{H}+\mathrm{L})$ (Invitrogen, USA, A32790), A594-conjugated donkey anti-mouse IgG(H+L) (Invitrogen, USA, A32744), and A594-conjugated goat antiMouse IgG1(Invitrogen, USA, A21125). All the antibodies were diluted to the working concentration recommended by the manufacturer. The primary antibodies were incubated overnight at $4{ }^{\circ} \mathrm{C}$ while the secondary antibodies were incubated at room temperature for 1 hour avoiding direct light. Finally, 4-6-diamidino-2-phenylindole (DAPI) at a concentration of $10 \mu \mathrm{g} / \mathrm{mL}$ were used to stain the nucleus. PBST washing for 5 minutes each was used between each step of this process. Photographs of stained images were taken using Zeiss instruments (Zeiss Germany).

\section{Celigo Image Cytometer instrumentation, data acquisition, and analysis}

In several related experiments, a Celigo Image Cytometer instrument has been used for high-throughput cell-based assays (17-19). In brief, a transmission and epifluorescence optical setup for one bright-field (BF) and four fluorescence (FL) imaging channels (Blue, Green, Red, and Far Red) were included and considered optional for analysis. The fluorescence filter was set for the corresponding colors as follows: Blue (EX: 377/50 nm, EM: 470/22 nm), Green (EX: 483/32 nm, EM: 536/40 nm), Red (EX: 531/40 nm, EM: 629/53 nm), and Far Red (EX: 628/40 nm, EM: 688/31 nm). Auto and manual focus were provided, with which the focus could be found in BF. After focus was registered, highly uniform images of entire well plates $(6,12,24$ and 96 wells) of different channels could be rapidly captured.

Before imaging, preparation including plate washing, fixing, permeabilizing, sealing, and antibody and fluorescein incubating similar to the process of immunofluorescence staining were made in wells. Subsequently, sample plates with stained cells treated differently were placed into the instrument for a new scan. This began with parameter setting for focus and exposure times for different channels, and then the candidate wells were selected for scanning. After scanning, analysis could be made based on the images taken. The software used allows different patterns of selected channels for analysis. In this study, we adopted "Target $1+$ Mask", in which we chose a nucleus with DAPI to identify a cell and to be the mask channel, and parameters of DAPI were set as 4 for as the Intensity Threshold, $10 \mu \mathrm{m}$ for Cell Diameter, and $10 \mu \mathrm{m}$ for the Dilation Radius to enlarge the calculated area. As we defined the actin-granule positive rate, we moved to "Gate" function provided by the software. A histogram of DAPI was made to include all cells for analysis, and a plot graph including the fluorescence data of Red ( $\alpha$-SMA) and Green (Vimentin) similar to flow cytometry (FCM) was made to assist creating a gate to determine whether a cellular actin-granule positive or negative was present.

\section{Flow cytometry and apoptosis analysis}

Fibroblasts were harvested by Ethylene Diamine Tetraacetic Acid (EDTA)-trypsin digestion, washed with PBS, stained with Propidium Iodide (PI, red, China, 4abio, Annexin V-Alexa Fluor488 /PI Apoptosis Detection Kit) and A488 conjugated anti-Annexin V antibody (green, China, 4abio, Annexin V-Alexa Fluor488/PI Apoptosis Detection Kit), then subject to flow cytometry for apoptosis analysis.

\section{Western Blot analysis}

Fibroblasts lysates were subjected to $\mathrm{WB}$ analysis to survey changes to their Col I, GEF-H1, p-GEF-H1, and $\alpha$-SMA under different circumstances. As the landmark molecules of fibrosis and ROCK signaling, Col I, GEF-H1, p-GEF-H1, and $\alpha$-SMA were separated from the lysates, transferred to PVDF film, and determined by chemiluminescence. The employed antibodies included rabbit anti- $\alpha$-Tubulin antibody (Proteintech, China, 11224-1-AP), rabbit antiCollagen I antibody (Proteintech, China, 14795-1-AP), rabbit monoclonal [E184] to alpha smooth muscle Actin antibody (Abcam, UK, ab32575), mouse monoclonal [1A4] to alpha smooth muscle Actin antibody(Abcam, UK, ab7817), rabbit monoclonal [EPR18134] to RhoA antibody (Abcam, UK, ab187027), rabbit monoclonal [EPR17963] to GEF H1-C-terminal antibody (Abcam, UK, ab201687), and rabbit monoclonal to Phospho-GEF-H1 (Ser886) antibody (CST, USA, 14143). 


\section{Statistical analysis}

Results are presented as mean \pm standard deviation. Groups were compared by analysis of variance and the student $t$ test, and a $\mathrm{P}$ value less than 0.05 was recognized as the statistically significant.

\section{Results}

\section{An individual phase of myofibroblast differentiation}

We isolated and cultured cardiac fibroblasts, which were identified by immunofluorescent staining (Figure 1A). As the differentiation from cardiac fibroblast to myofibroblast could be induced by FCS, profibrotic cytokines autocrine/ paracrine, and physical injury from passaging, we then inspected the phenotype of Passage 1 (P1) and Passage 2 (P2) cells and found that myofibroblasts, with a limited percentage (around 15\%) in P1, accounted for more than $40 \%$ in $\mathrm{P} 2$ (Figure $1 B$ ) (20-22). To verify this difference between P1 and P2 cells, Western Blot (WB) was employed to compare their Col-I and $\alpha$-SMA expression. However, WB results demonstrated the upregulation of Col-I in P2 and an unexpected high level of $\alpha$-SMA in P1 (Figure 1C). To clarify the discordance of Col-I and $\alpha$-SMA expression, the phenotype identification of $\mathrm{P} 1$ was retrospectively performed, revealing that in a number of P1 cells $\alpha$-SMA was also synthesized. In addition, rather than being assembled into a filament immediately, these monomers were condensed as a granule into a perinuclear hollow in the vimentin (intermediate filament) network (Figure 1B).

To examine these granules in more detail, we again captured several fibroblasts with unassembled $\alpha$-SMA after incubation, as well as myofibroblasts with actin-bundles (Figure $2 A$ ). The unassembled $\alpha$-SMA was seen to be different from actin filaments through the cell connecting focal adhesions and was condensed into a granule with higher fluorescence intensity than actin filaments. In addition, it was always located in a circular hollow in the vimentin network and near the nucleus to which it was usually notched (Figure 2B).

\section{Ang II induces myofibroblast differentiation and GEF-H1 Ser886 phosphorylation}

As we identified the actin granule rather than assembled filament was quite common in P1 fibroblasts, we presumed ROCK signaling which regulates stress actin fiber assembly might play a possible role in myofibroblast differentiation.
To verify its involvement, myofibroblast differentiation was induced by Ang II in P1 cells (Figure 3A). Rather than the conventional 24 hours, the incubation time was narrowed down to 8 hours to reveal the early phase of differentiation. Subsequently, WB was employed to investigate Collagen I, $\alpha$-SMA, p-GEF-H1, and GEF-H1changes after Ang II induction, resulting in a unanimous increase (Figure $3 B$ ). This suggested Ang II could upregulate Collagen I and the phosphorylation of GEF-H1 on Ser886 to activate the ROCK signaling pathway.

\section{Fasudil prevents actin fiber assembly and myofibroblast differentiation by blocking ROCK signaling}

On the basis of our discovery of the actin-granule phase of myofibroblast differentiation and that p-GEF-H1, GEF-H1, and $\alpha$-SMA could be upregulated by Ang II, we hypothesized that the HDAC6-acetylated $\alpha$-tubulin deacetylation-GEF-H1-ROCK signaling axis played a role in the process and could potentially be blocked to prevent it. The HDAC6 inhibitor Ricolinostat and ROCK inhibitor Fasudil were then applied, however in contrast to Ricolinostat, Fasudil apparently reshaped the outlook of cardiac fibroblasts, raising concern about its apoptosis induction capability (Figure $4 A$ ). Therefore, we performed CCK- 8 and flow cytometry analysis and found that while $50 \mu \mathrm{M}$ Fasudil did alter the shape of cells, it did not threaten their survival $(90 \%$ free from death and early apoptosis) (Figure 4B). In reference to the unexpected WB results of Col I, Ricolinostat failed to prevent the enhanced expression of Col I induced by Ang II. However, Fasudil, which is supposed to have influences on downstream effectors, succeeded to limit Col I upregulation (Figure 4C). With regard to Rho A and GEF-H1 quantity and GEF-H1 phosphorylation as other factors affecting ROCK signaling, we found that Fasudil, rather than Ricolinostat significantly diminished RhoA, GEF-H1, and phosphorylated GEF-H1 demolishing the ROCK signaling pathway (Figure 4C).

Immunofluorescent staining was again utilized to investigate the effects of Ricolinostat and Fasudil at the cellular level. In cells without Ang II incubation, Ricolinostat and Fasudil evidently increased the proportion of fibroblasts with actin-granules. In addition, Fasudil still prevented actin assembly after Ang II incubation; in contrast to Ricolinostat which again failed to attenuate Ang II-induced actin assembly (Figure $5 A$ ). To quantify this difference, actin-granule positive rates were determined by Celigo image cytometer. As actin-granules were always 
A
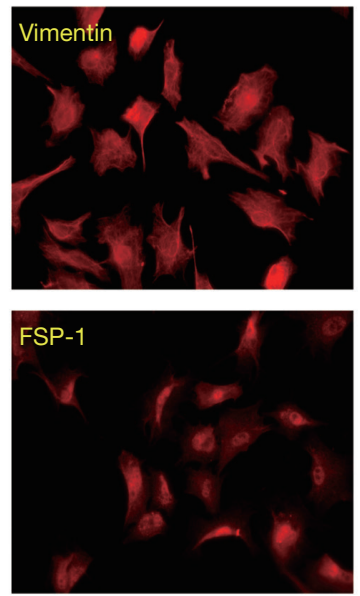

B
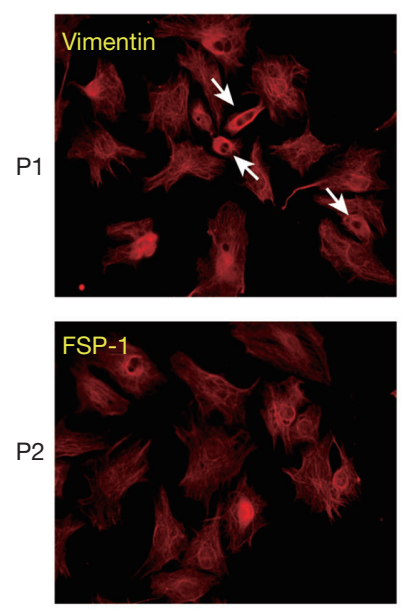
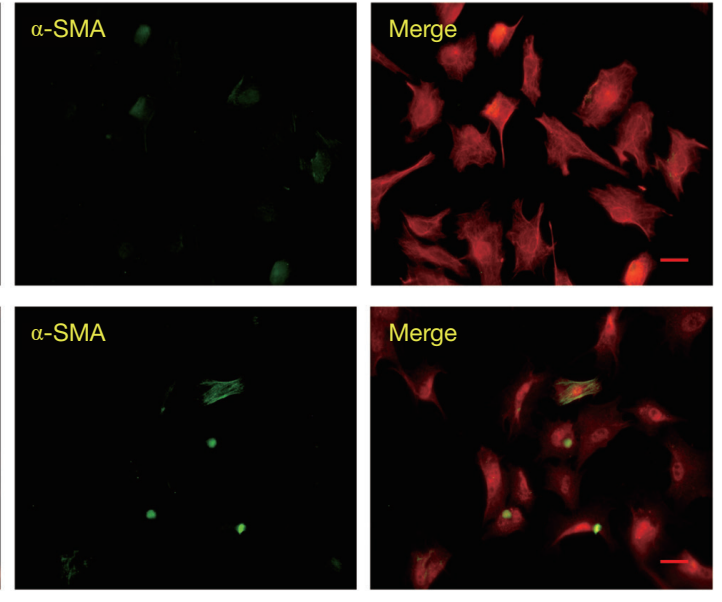

$\alpha-S M A$

$\alpha$-tubulin

C

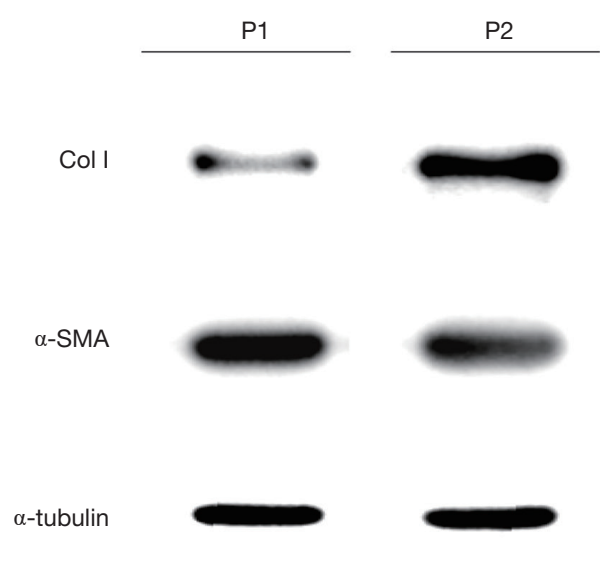

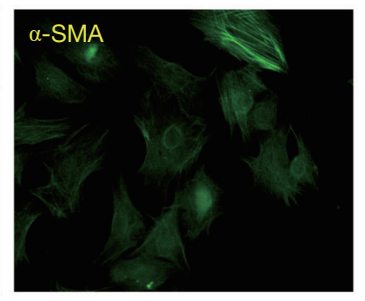
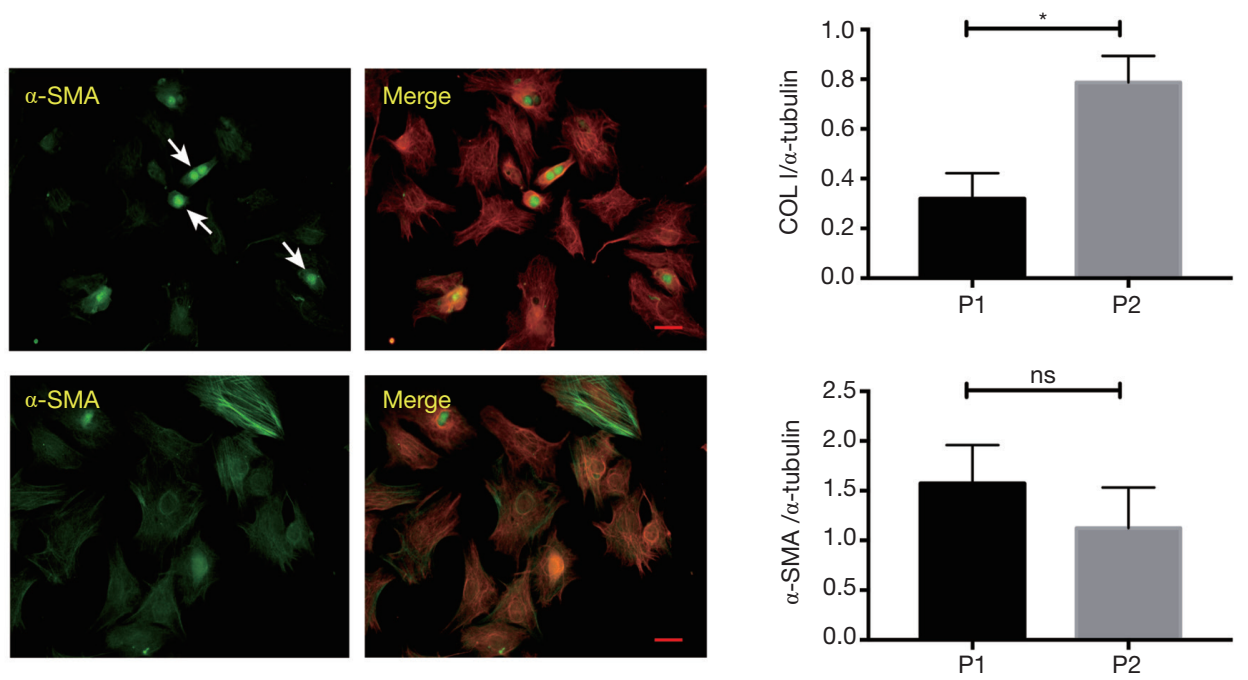

Figure 1 Myofibroblast differentiation could be seen in P2 while $\alpha$-SMA synthesis occurred in P1. (A) Immunofluorescent staining of Vimentin (upper panel, red), fibroblast-specific protein-1 (FSP-1) (lower panel, red), and $\alpha$-SMA (green) for phenotype identification of fibroblasts isolated from neonatal rats. Scale bar $50 \mu \mathrm{m}$. (B) Immunofluorescent staining of Vimentin (red) and $\alpha$-SMA (green). Scale bar $50 \mu \mathrm{m}$. Cells after the first passage (upper panel) and second passage (lower panel) were compared and showed that myofibroblasts with actin filaments and of irregular shapes could be seen in P2 cells while a number of P1 cells maintain the fibroblast-specific shuttle shape with $\alpha$-SMA synthetized and condensed into the perinuclear region. (C) Representative Western blot gel depicts the different levels of Col I and $\alpha$-SMA in $\mathrm{P} 1$ and $\mathrm{P} 2$ cells. The mean value of the protein expression level of Col-1 and $\alpha$-SMA in P1 and P2 cells. Compared to P2, Col I expression was limited in P1 cells while $\alpha$-SMA was with similar concentration $\left(^{*}, \mathrm{P}<0.05, \mathrm{n}=3\right)$. Arrows indicate actin granule in vimentin hollow.

placed in the peri-nuclear region, the number of cells was counted by reference to the nucleus with DAPI, while the calculated area of the nucleus appeared bigger than it really was. Accordingly, $\alpha$-SMA was then detected and marked as (actin-granule) positive (Figure S1). With or without Ang II, the addition of Fasudil led to an increase in the positive rate to more than $40 \%$. However, the percentage of cells pre-treated with Ricolinostat was drawn back by Ang II from over 40 percent to 20 percent (Figure 5B), which is consistent with the effects of both on GEF-H1 phosphorylation and Col I upregulation. Together this demonstrated that Fasudil abrogated the ROCK signaling 
A

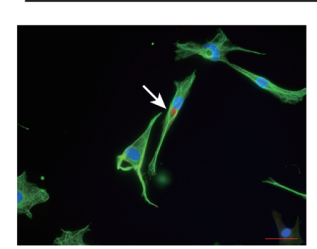

B

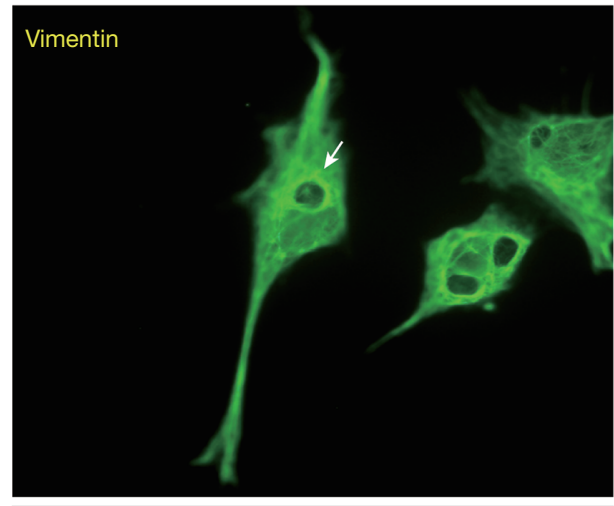

DAPI

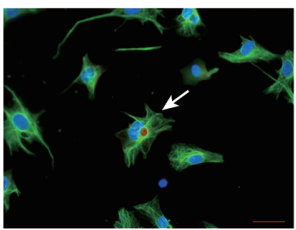

Actin-granule

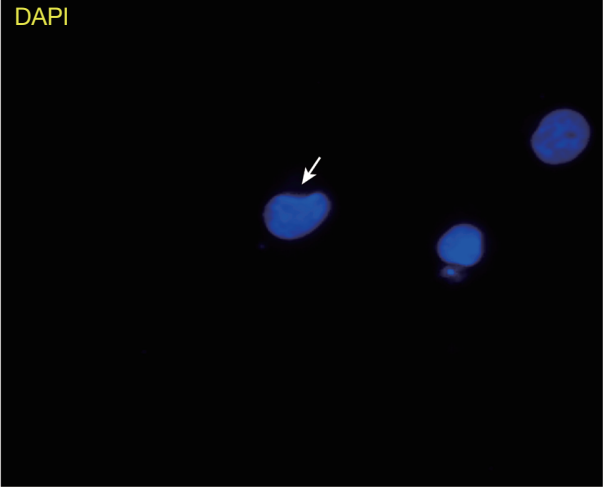

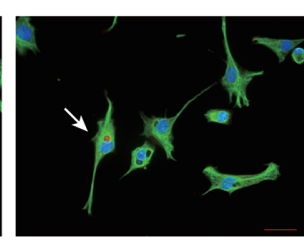

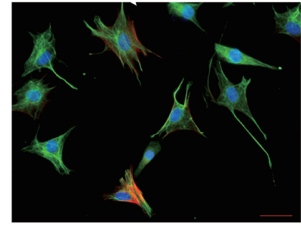

a-SMA

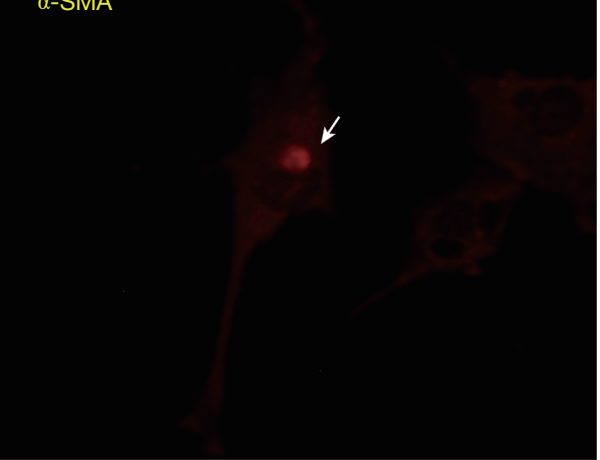

Merge

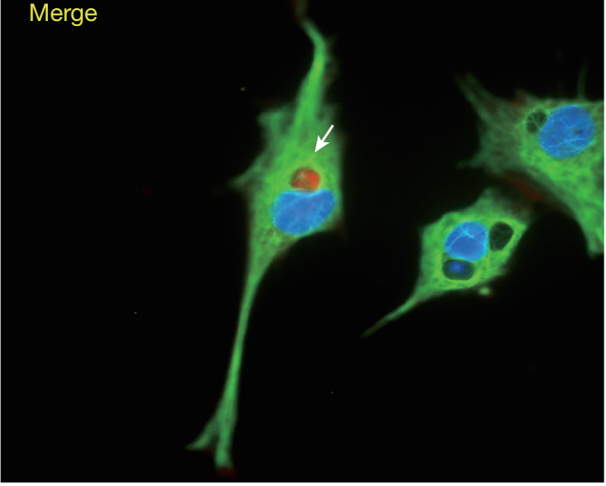

Figure 2 Actin granule formed before cardiac myofibroblast differentiation. (A) Immunofluorescent staining of Vimentin (green), alphasmooth muscle actin ( $\alpha$-SMA) (red), and nucleus (DAPI, blue). Scale bar $50 \mu \mathrm{m}$. Unassembled $\alpha$-SMA was condensed into a granule with higher fluorescence intensity than actin filaments. (B) Immunofluorescent staining of Vimentin (green), alpha-smooth muscle actin ( $\alpha$-SMA) (red), and nucleus (DAPI, blue). Magnification $\times 400$. Actin granule is always located in a circular hollow in the vimentin network and beside the notched nucleus. Arrows indicate actin granule located in vimentin hollow besides cell nucleus.

pathway from multiple levels and neutralized Ang II induction, by which myofibroblast differentiation could be obstructed at the actin-granule phase and Col I upregulation suspended thereafter.

\section{Discussion}

Pathological fibrosis is highly relevant to $\mathrm{AF}$ as it could stabilize re-entrant drivers leading to the perpetuation of this arrhythmia (23). Many mechanisms have been shown to promote fibrosis in AF, including calcium overload, oxidative stress, inflammation, and RAAS activation (24-29) with fibroblasts playing a central role in each. The primary function of fibroblasts is to maintain ECM homeostasis and sustain myocytes, while in AF their fibrotic potency increases significantly leading to atrial fibrosis (29). While the differentiation of cardiac myofibroblasts is the most important cellular mechanism in atrial fibrosis and remodeling, in addition to cardiac fibroblasts, myofibroblasts could be derived from epithelial cells, endothelial cells, and circulating fibrocytes (30). Profibrotic and pro-inflammatory cytokines including TGF- $\beta 1$, Ang II, and IL-6 have been documented as initiators of this differentiation. 
A
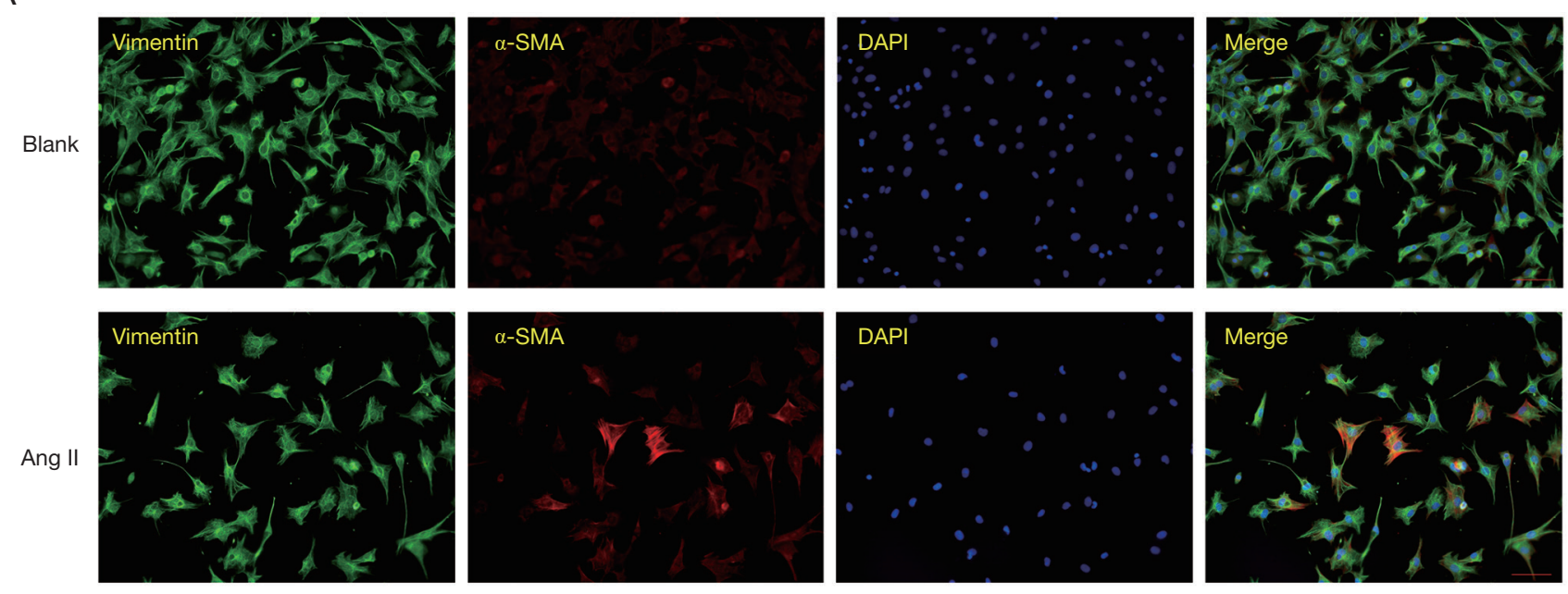

B
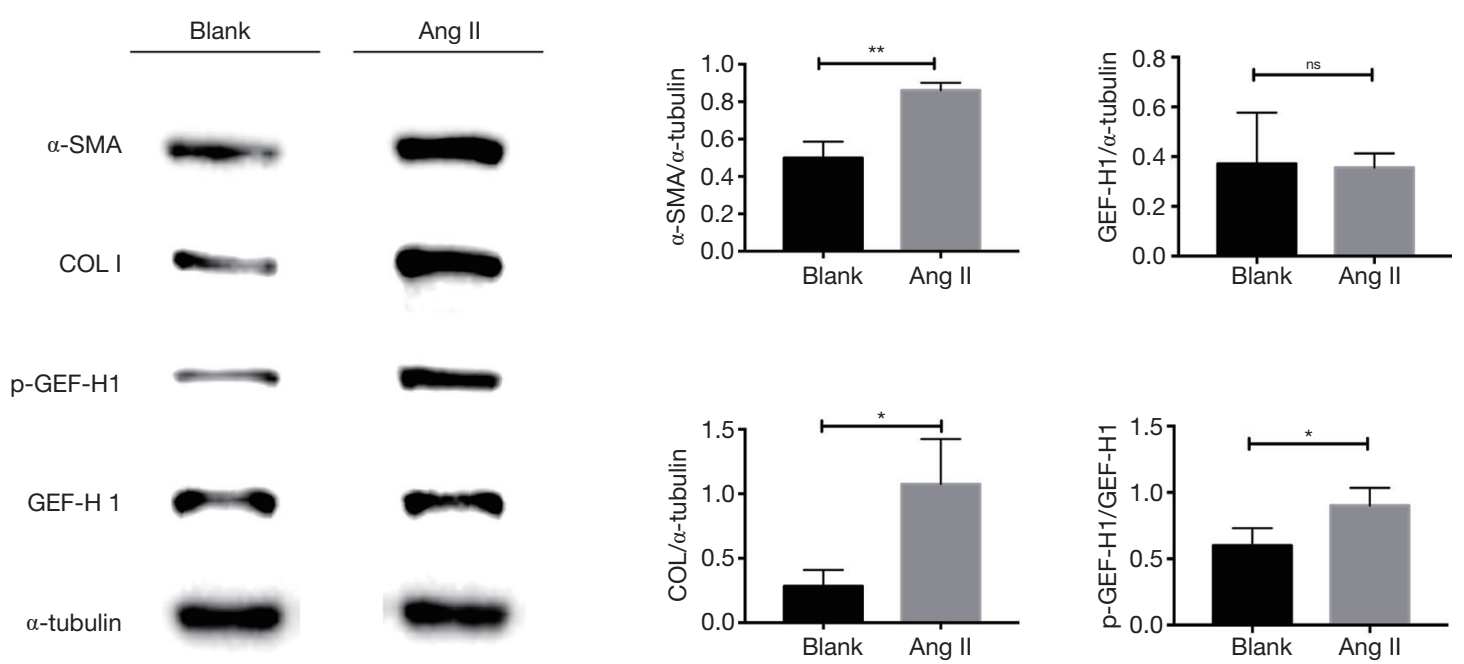

Figure 3 Ang II upregulated Collagen I, $\alpha$-SMA, and p-GEF-H1 and induced myofibroblast differentiation. (A) Immunofluorescent staining of Vimentin (green), $\alpha$-SMA (red), and nucleus (DAPI, blue). Scale bar $100 \mu \mathrm{m}$. Angiotensin II (Ang II) incubation for 8 hours successfully induced myofibroblast differentiation in some P1 cells. (B) Representative Western blot images demonstrate the different levels of Collagen I, $\alpha$-SMA, GEF-H1, and p-GEF-H1 in P1 activated by Ang II for 8 hours or not. The mean value of the protein expression level of Collagen I, $\alpha$-SMA, GEF-H1, and p-GEF-H1 in cells treated under the same conditions ${ }^{*}, \mathrm{P}<0.05$; ${ }^{* *}, \mathrm{P}<0.01, \mathrm{n}=3$ ).

The two prominent characters of myofibroblasts are the formation of actin filaments and upregulation of fibrotic capability. As $\alpha$-SMA expression is not seen in the original status of cardiac fibroblasts, it is treated as a marker of myofibroblast differentiation (31). In this study, we described the conflicting phenomenon of limited Col I and significantly increased $\alpha$-SMA in P1 cells and discovered an individual phase of actin-granule formation settled in myofibroblast differentiation. As cells in the actin- granule phase maintain their shuttle shape and barren Col I expression, it is now debatable whether a simple definition of myofibroblast differentiation with the occurrence of $\alpha$-SMA synthesis is still acceptable. Moreover, few studies have addressed the relationship between the $\alpha$-SMA and fibrosis aspect of myofibroblast differentiation in the past because an intermediate step was assumed not to be present. In addition to discovering this special phase, we also revealed a proportion (around 25\%) of P1 cells were 

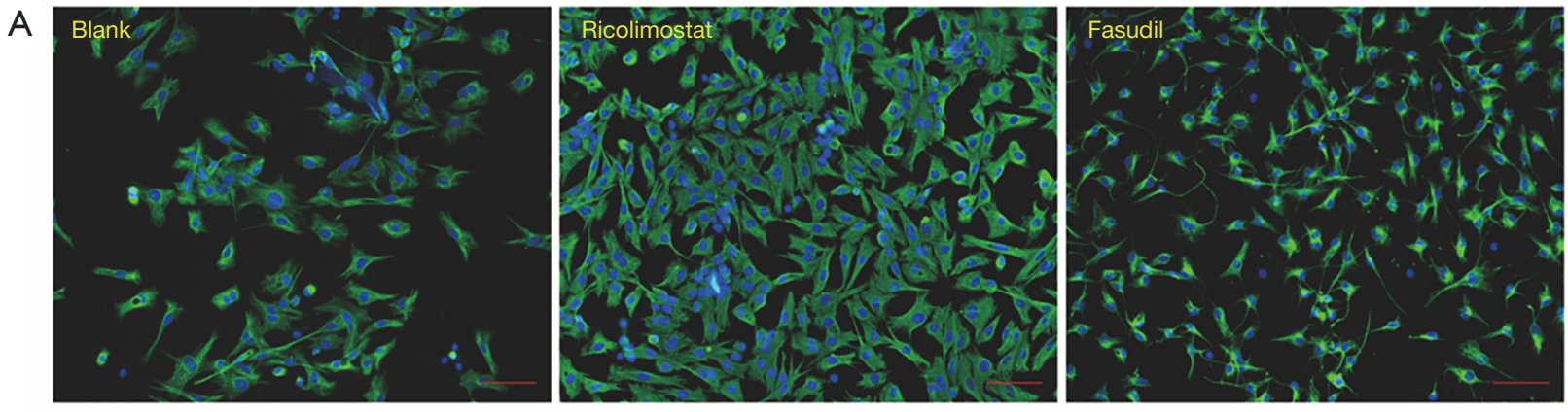

B
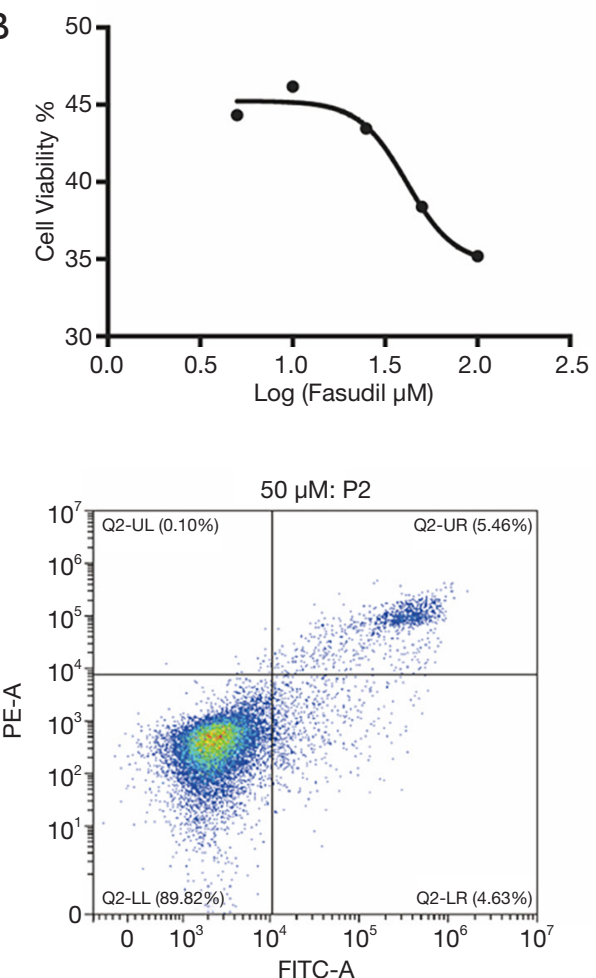

C

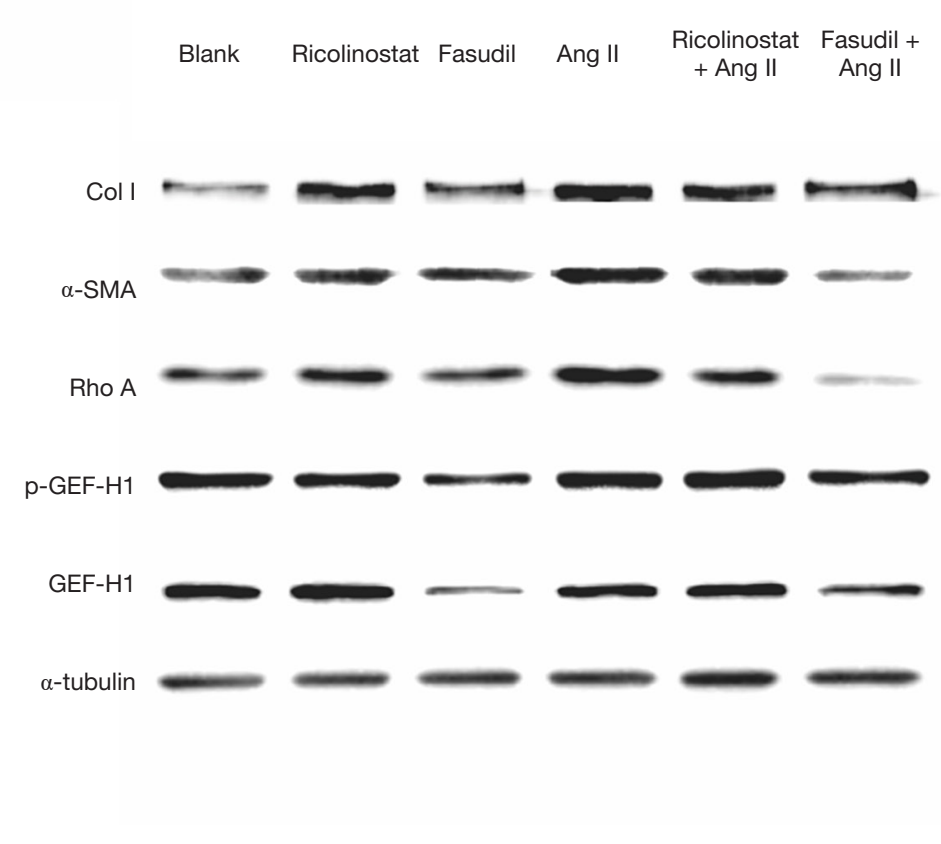

Figure 4 Fasudil abrogated Ang II induced ROCK signaling activation and Col I upregulation. (A) Immunofluorescent staining of Vimentin (green), and nucleus (DAPI, blue). Scale bar $100 \mu \mathrm{m}$. P1 cells incubated with Fasudil for 24 hours were reshaped significantly, while Ricolinostat increased the intensity of the vimentin network. (B) CCK-8 analysis (upper panel) and flow cytometry analysis (lower panel) of Fasudil's effects on fibroblast viability. (C) Representative Western blot gel depicts the protein expression of Col I, p-GEF-H1, and GEF-H1. Cells were treated with Fasudil, Ricolinostat, AngII, Fasudil + Ang II, and Ricolinostat + Ang II for 24 hours. Fasudil and Ricolinostat were added 6 hours before the addition of Ang II.

in this phase, suggesting it may be of sufficient duration for us to block F-actin assembly and estimate its effect on Col I expression.

The concept of stress actin fibers has a long history and proposes that in response to stress, actin filaments may form in non-muscle cells, and when this occurs in fibroblasts, three different categories-dorsal fibers, ventral fibers and transvers arcs may form $(32,33)$. First formed are the dorsal fibers as short F-actins oriented from focal adhesion and inserted by actinin. Then the bundles were elongated by the assembly of more F-actins and myosin clusters to form transverse arcs. At last, the ventral stress fibres as the most common were realized by the free F-actin tip blocked by another focal adhesion. The core of focal adhesion is the 
A

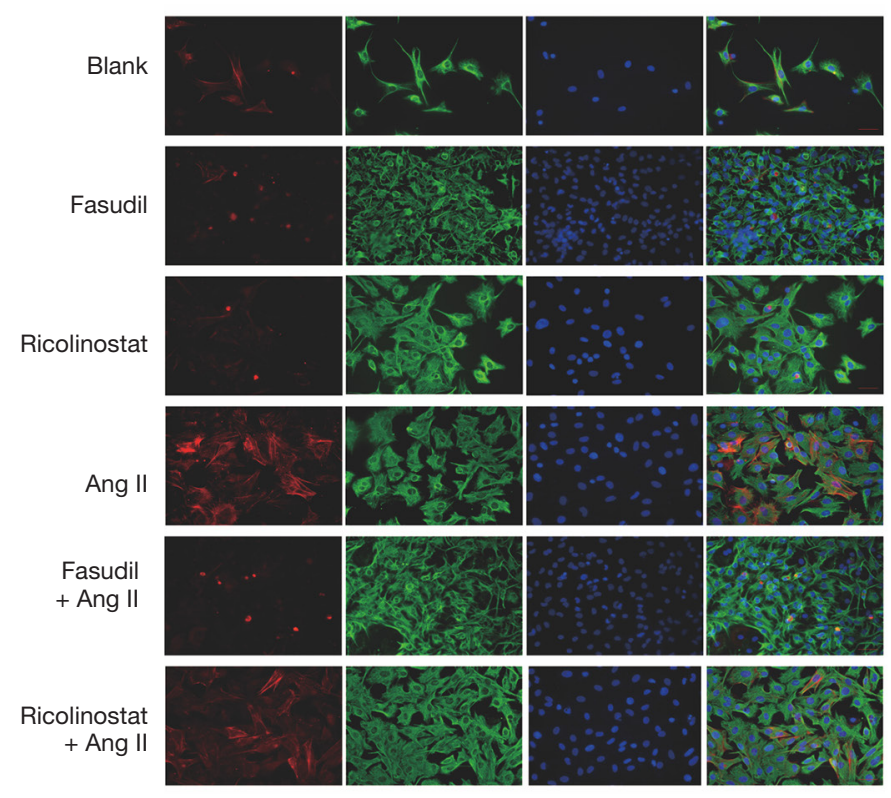

B

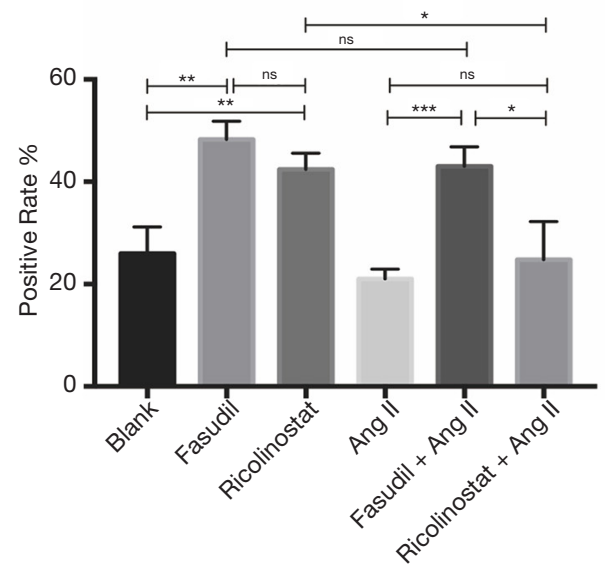

Figure 5 Fasudil prevented Ang II induced actin filament assembly and myofibroblast differentiation. (A) Immunofluorescent staining of Vimentin (green), $\alpha$-SMA (red) and nucleus (blue). Scale bar $50 \mu \mathrm{m}$. Cells were treated with Fasudil, Ricolinostat, Ang II, Fasudil + Ang II, and Ricolinostat + Ang II for 24 hours. Fasudil and Ricolinostat were added 6 hours before the addition of Ang II. Fasudil and Ricolinostat both attenuated actin filaments assembly, while only Fasudil could prevent Ang II induced F-actin assembly and myofibroblast differentiation. (B) Whole view analysis of actin-granule positive rate by Celigo image cytometer. Mean value of actin-granule positive rate of cells treated with the same conditions as $\mathrm{A}\left({ }^{*}, \mathrm{P}<0.05 ;{ }^{* *}, \mathrm{P}<0.01 ;{ }^{* * *}, \mathrm{P}<0.001, \mathrm{n}=3\right)$.

integrins constituted by heterodimers of $\alpha 5 \beta 1$ or $\alpha \mathrm{v} \beta 3$. On the external surfaces integrins bind to integrin receptors clinging on to fibronectin, and on the cytoplasmic surfaces assembled is the focal adhesion complex with both integrininteracting and actin-interacting domains.

In cardiac research, myofibroblast differentiation is widely accepted to describe the formation of microfilaments in cardiac fibroblasts, which seems to be another description of a stress actin fiber (especially for ventral fibers) assembly. Despite the different contraction modes of different stress fiber kinds, most researchers agree that stress actin fibers could increase the contraction and migration capability of non-muscle cells (34-36). ROCK signaling is essential for stress actin fiber assembly, of which ROCK-1 and ROCK-2 are the main effectors phosphorylating myosin light chain 2 and promoting stress fiber formation, which in turn is activated by small GTPase RhoA $(26,37)$. Additionally, cross regulation is a common phenomenon between cytoskeleton departments. Rho-specific GEF-H1 is normally fixed to microtubules, and when microtubule stability is impaired it will be released to activate Rho $A$ and ROCK signaling. In addition, overexpression of
GEF-H1 will give rise to deacetylation and instability of microtubules. However, unlike the release and overexpression of GEF-H1 which simply facilitate stress actin fiber assembly, the effect of GEF-H1 phosphorylation seems to be controversial. Yamahashi et al. demonstrated that PAR1b induces phosphorylation of GEF-H1 at Ser885 and Ser959 inhibiting its RhoA-specific GEF activity. In contrast, phosphorylation at Ser886 by vimentin depletion will increase its activity to RhoA15 (38). Accordingly, $\alpha$-SMA expression, F-actin assembly, and GEF-H1 phosphorylation at Ser886 were detected by us after Ang II activation.

Recently HDAC6 has been verified to deacetylate acetylated tubulin and disrupt cardiomyocyte microtubule system. We also found that HDAC 6 was elevated in the left atrial appendage of AF patients (data no shown) (14). In Staphylococcus aureus infection, GEF-H1 activity was blocked by HDAC6 down-regulation, and HDAC6 knockout and inhibition diminished ROCK-induced vascular leakage and inflammation (39). As a selective inhibitor of HDAC6, Ricolinostat has been proven to be of value attenuating oxidative stress to diminish inflammation and inducing apoptosis to inhibit tumor growth through various signaling 
pathways (40-43). However, in this study Ricolinostat failed to prevent Ang II induced myofibroblast differentiation (a process accompanied by fibroblasts proliferation) and was discovered to promote GEF-H1 phosphorylation on Ser886 with enhanced activity of RhoA. We also observed the intensity of the vimentin network to be increased by Ricolinostat. Even though vimentin depletion could activate the ROCK signaling pathway, the effect of its overexpression on GEF-H1 activity and the ROCK signaling pathway remains unclear (15). Additionally, we also employed the ROCK inhibitor Fasudil in our study. Santos et al. have reported Fasudil suppresses cardiac myofibroblast function and Shimizu et al. have addressed the possible correlation between the ROCK signaling pathway and pathologic cardiac fibrosis. However, in the present study we found, for the first time, that Fasudil could downregulate GEF-H1 expression and its phosphorylation on Ser886, through which it abrogated the ROCK signaling pathway, actin filament assembly, and pro-fibrotic potency upregulation of myofibroblast differentiation $(44,45)$.

\section{Acknowledgments}

Funding: This work was supported by the National Natural Science Foundation of China (81900311 from HSC, 81970345 from HFT) and Science and Technology Agency Foundation of Sichuan province (2019YFS0347 and 2019YFS0353).

\section{Footnote}

Reporting Checklist: The authors have completed the ARRIVE reporting checklist. Available at http://dx.doi. org/10.21037/atm-20-8231

Data Sharing Statement: Available at http://dx.doi. org/10.21037/atm-20-8231

Conflicts of Interest: All authors have completed the ICMJE uniform disclosure form (available at http://dx.doi. org/10.21037/atm-20-8231). The authors have no conflicts of interest to declare.

Ethical Statement: The authors are accountable for all aspects of the work in ensuring that questions related to the accuracy or integrity of any part of the work are appropriately investigated and resolved. The experiments were supervised by Animal Ethics Committee of West
China Hospital and the Research Core Facility of West China Hospital, in compliance with the guidelines of Ethics Committee of West China Hospital for the care and use of animals.

Open Access Statement: This is an Open Access article distributed in accordance with the Creative Commons Attribution-NonCommercial-NoDerivs 4.0 International License (CC BY-NC-ND 4.0), which permits the noncommercial replication and distribution of the article with the strict proviso that no changes or edits are made and the original work is properly cited (including links to both the formal publication through the relevant DOI and the license). See: https://creativecommons.org/licenses/by-nc-nd/4.0/.

\section{References}

1. Wolf PA, Abbott RD, Kannel WB. Atrial fibrillation as an independent risk factor for stroke: the Framingham Study. Stroke 1991;22:983-8.

2. Go AS, Hylek EM, Phillips KA, et al. Prevalence of diagnosed atrial fibrillation in adults: national implications for rhythm management and stroke prevention: the AnTicoagulation and Risk Factors in Atrial Fibrillation (ATRIA) Study. JAMA 2001;285:2370-5.

3. Miyasaka Y, Barnes ME, Gersh BJ, et al. Secular trends in incidence of atrial fibrillation in Olmsted County, Minnesota, 1980 to 2000, and implications on the projections for future prevalence. Circulation 2006;114:119-25.

4. Hinz B. Formation and function of the myofibroblast during tissue repair. J Invest Dermatol 2007;127:526-37.

5. Wachtell K, Lehto M, Gerdts E, et al. Angiotensin II receptor blockade reduces new-onset atrial fibrillation and subsequent stroke compared to atenolol: the Losartan Intervention For End Point Reduction in Hypertension (LIFE) study. J Am Coll Cardiol 2005;45:712-9.

6. Bhuriya R, Singh M, Sethi A, et al. Prevention of recurrent atrial fibrillation with angiotensin-converting enzyme inhibitors or angiotensin receptor blockers: a systematic review and meta-analysis of randomized trials. J Cardiovasc Pharmacol Ther 2011;16:178-84.

7. Nattel S. Molecular and cellular mechanisms of atrial fibrosis in atrial fibrillation. JACC Clin Electrophysiol 2017;3:425-35.

8. Travers JG, Kamal FA, Robbins J, et al. Cardiac fibrosis: the fibroblast awakens. Circ Res 2016;118:1021-40.

9. Burridge K, Wittchen ES. The tension mounts: stress 
fibers as force-generating mechanotransducers. J Cell Biol 2013;200:9-19.

10. Barnes WG, Reiter E, Violin JD, et al. beta-Arrestin 1 and Galphaq/11 coordinately activate RhoA and stress fiber formation following receptor stimulation. J Biol Chem 2005;280:8041-50.

11. Aoki H, Izumo S, Sadoshima J. Angiotensin II activates RhoA in cardiac myocytes: a critical role of RhoA in angiotensin II-induced premyofibril formation. Circ Res 1998;82:666-76.

12. Leung T, Manser E, Tan L, et al. A novel serine/threonine kinase binding the Ras-related RhoA GTPase which translocates the kinase to peripheral membranes. J Biol Chem 1995;270:29051-4.

13. Zhang D, Hu X, Henning RH, et al. Keeping up the balance: role of HDACs in cardiac proteostasis and therapeutic implications for atrial fibrillation. Cardiovasc Res 2016;109:519-26.

14. Zhang D, Wu C.T, Qi X, et al. Activation of histone deacetylase-6 induces contractile dysfunction through derailment of alpha-tubulin proteostasis in experimental and human atrial fibrillation. Circulation 2014;129:346-58.

15. Jiu Y, Peranen J, Schaible N, et al. Vimentin intermediate filaments control actin stress fiber assembly through GEF-H1 and RhoA. J Cell Sci 2017;130:892-902.

16. Krendel M, Zenke FT, Bokoch GM. Nucleotide exchange factor GEF-H1 mediates cross-talk between microtubules and the actin cytoskeleton. Nat Cell Biol 2002;4:294-301.

17. Kessel S, Cribbes S, Dery O, et al. High-Throughput 3D Tumor Spheroid Screening Method for Cancer Drug Discovery Using Celigo Image Cytometry. SLAS Technol 2017;22:454-65.

18. Chan LL, Smith T, Kumph KA, et al. A high-throughput AO/PI-based cell concentration and viability detection method using the Celigo image cytometry. Cytotechnology 2016;68:2015-25.

19. Zhang H, Chan LL, Rice W, et al. Novel high-throughput cell-based hybridoma screening methodology using the Celigo Image Cytometer. J Immunol Methods 2017;447:23-30.

20. Xu X, Pang J, Yin H, et al. Hexarelin suppresses cardiac fibroblast proliferation and collagen synthesis in rat. Am J Physiol Heart Circ Physiol 2007;293:H2952-8.

21. Dorn LE, Petrosino JM, Wright P, et al. CTGF/CCN2 is an autocrine regulator of cardiac fibrosis. J Mol Cell Cardiol 2018;121:205-11.

22. Lee AA, Dillmann WH, McCulloch AD, et al. Angiotensin II stimulates the autocrine production of transforming growth factor-beta 1 in adult rat cardiac fibroblasts. J Mol Cell Cardiol 1995;27:2347-57.

23. Zahid S, Cochet H, Boyle PM, et al. Patient-derived models link re-entrant driver localization in atrial fibrillation to fibrosis spatial pattern. Cardiovasc Res 2016;110:443-54.

24. Martins R.P, Kaur K, Hwang E, et al. Dominant frequency increase rate predicts transition from paroxysmal to long-term persistent atrial fibrillation. Circulation 2014;129:1472-82.

25. Wang L, Myles RC, De Jesus NM, et al. Optical mapping of sarcoplasmic reticulum $\mathrm{Ca} 2+$ in the intact heart: ryanodine receptor refractoriness during alternans and fibrillation. Circ Res 2014;114:1410-21.

26. Sun S, Kee HJ, Jin L, et al. Gentisic acid attenuates pressure overload-induced cardiac hypertrophy and fibrosis in mice through inhibition of the ERK1/2 pathway, J Cell Mol Med 2018;22:5964-77.

27. Friedrichs K, Baldus S, Klinke A. Fibrosis in Atrial Fibrillation - Role of Reactive Species and MPO. Front Physiol 2012;3:214.

28. Harada M, Van Wagoner DR, Nattel S. Role of inflammation in atrial fibrillation pathophysiology and management. Circ J 2015;79:495-502.

29. Huang Z, Chen XJ, Qian C, et al. Signal Transducer and Activator of Transcription 3/MicroRNA-21 Feedback Loop Contributes to Atrial Fibrillation by Promoting Atrial Fibrosis in a Rat Sterile Pericarditis Model. Circ Arrhythm Electrophysiol 2016;9:e003396.

30. Krenning G, Zeisberg EM, Kalluri R. The origin of fibroblasts and mechanism of cardiac fibrosis. J Cell Physiol 2010;225:631-7.

31. Jalife J, Kaur K. Atrial remodeling, fibrosis, and atrial fibrillation. Trends Cardiovasc Med 2015;25:475-84.

32. Abercrombie M, Heaysman JE, Pegrum SM. The locomotion of fibroblasts in culture. IV. Electron microscopy of the leading lamella. Exp Cell Res 1971;67:359-67.

33. Pellegrin S, Mellor H. Actin stress fibres. J Cell Sci 2007;120:3491-9.

34. Hotulainen P, Lappalainen P. Stress fibers are generated by two distinct actin assembly mechanisms in motile cells. J Cell Biol 2006;173:383-94.

35. Tojkander S, Gateva G, Schevzov G, et al. A molecular pathway for myosin II recruitment to stress fibers. Curr Biol 2011;21:539-50.

36. Tee YH, Shemesh T, Thiagarajan V, et al. Cellular chirality arising from the self-organization of the actin cytoskeleton. 
Nat Cell Biol 2015;17:445-57.

37. Heasman SJ, Ridley AJ. Mammalian Rho GTPases: new insights into their functions from in vivo studies. Nat Rev Mol Cell Biol 2008;9:690-701.

38. Yamahashi Y, Saito Y, Murata-Kamiya N, et al. Polarityregulating kinase partitioning-defective $1 \mathrm{~b}$ (PAR1b) phosphorylates guanine nucleotide exchange factor $\mathrm{H} 1$ (GEF-H1) to regulate RhoA-dependent actin cytoskeletal reorganization. J Biol Chem 2011;286:44576-84.

39. Karki P, Ke Y, Tian Y, et al. Staphylococcus aureusinduced endothelial permeability and inflammation are mediated by microtubule destabilization. J Biol Chem 2019;294:3369-84.

40. Li L, Liu F, Huang W, et al. Ricolinostat (ACY-1215) inhibits VEGF expression via PI3K/AKT pathway and promotes apoptosis in osteoarthritic osteoblasts. Biomed Pharmacother 2019;118:109357.

41. Zhang WB, Yang F, Wang Y, et al. Inhibition of HDAC6 attenuates LPS-induced inflammation in macrophages

Cite this article as: He L, Liu R, Yue H, Ren S, Zhu G, Guo Y, Qin C. Actin-granule formation is an additional step in cardiac myofibroblast differentiation. Ann Transl Med 2021;9(2):165. doi: $10.21037 /$ atm-20-8231 by regulating oxidative stress and suppressing the TLR4MAPK/NF-kappaB pathways. Biomed Pharmacother 2019;117:109166.

42. García-Guerrero E, Gotz R, Doose S, et al. Upregulation of CD38 expression on multiple myeloma cells by novel HDAC6 inhibitors is a class effect and augments the efficacy of daratumumab. Leukemia 2021;35:201-14.

43. Vekaria PH, Kumar A, Subramaniam D, et al. Functional cooperativity of p97 and histone deacetylase 6 in mediating DNA repair in mantle cell lymphoma cells. Leukemia 2019;33:1675-86.

44. Shimizu T, Liao JK. Rho Kinases and Cardiac Remodeling. Circ J 2016;80:1491-8.

45. Santos GL, Hartmann S, Zimmermann WH, et al. Inhibition of Rho-associated kinases suppresses cardiac myofibroblast function in engineered connective and heart muscle tissues. J Mol Cell Cardiol 2019;134:13-28.

(English Language Editor: B. Draper) 


\section{Supplementary}
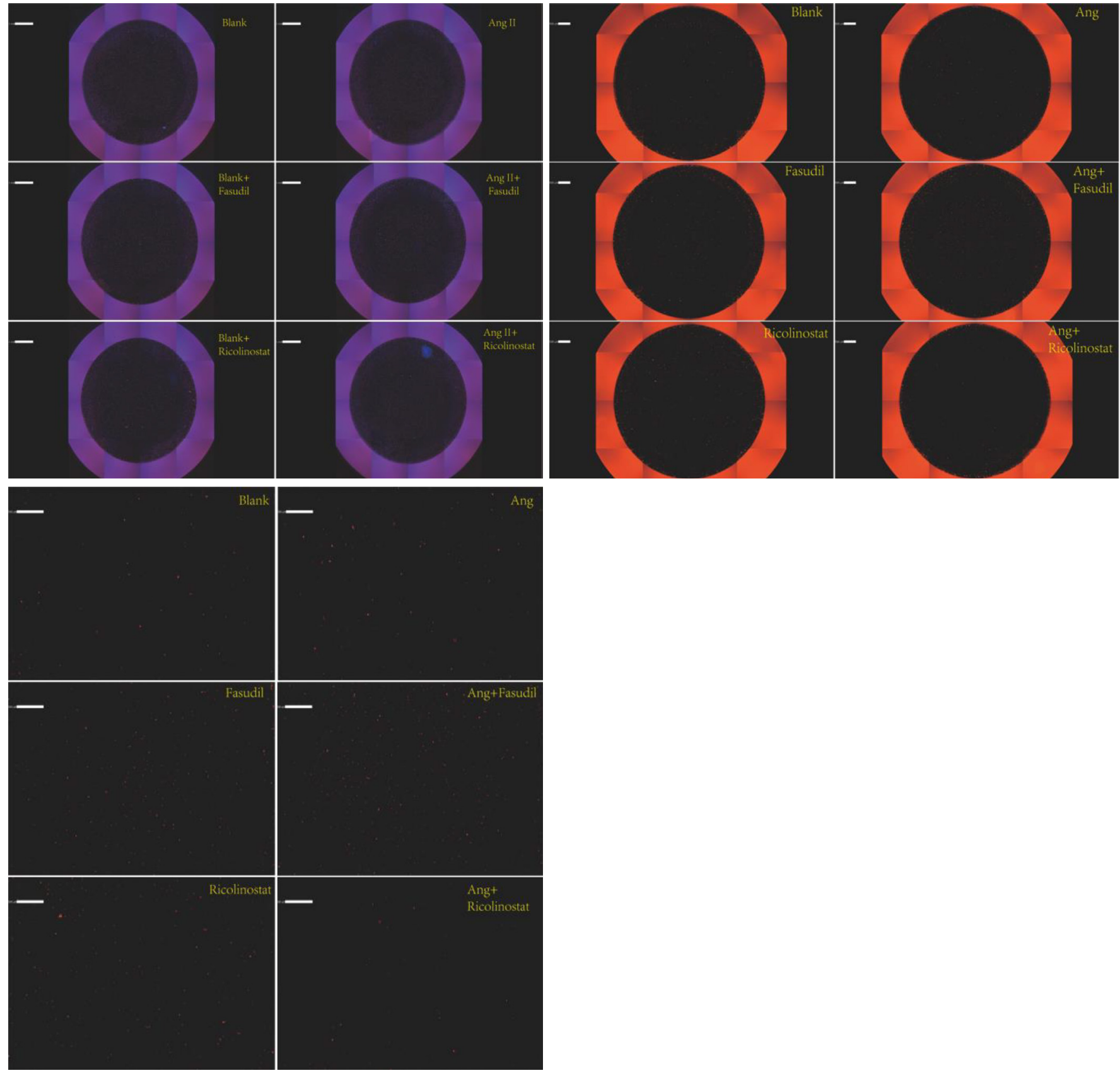

Figure S1 Celigo analysis of actin granule positive rate. 